\title{
Covid-19 pandemic implications from an addiction perspective
}

\author{
Naveen Kumar Dhagudu ${ }^{1 *}$, E. Omesh Kumar ${ }^{2}$ \\ Dept. of Psychiatry, ESIC Medical College, Telangana, India \\ *Corresponding Author: Naveen Kumar Dhagudu \\ Email: naveendhagudu@gmail.com
}

Sir,

People of the world are grappling with the pandemic coronavirus disease (COVID-19). Currently, as on May 10th, 2020, 05:30 GMT 3,884,434 people have been infected and 272,859 have died across the globe. ${ }^{2}$ Given this public health emergency, many countries implemented measures like lockdown, social distancing, social isolation and home quarantine etc as means of containing the disease and decreasing its fatality. These measures have led to huge financial losses to the public and governments. It has left us in a state where it is difficult to address other medical conditions of public health, most importantly those arising from the process containment measures. Psychological health issues like anxiety, depression, stress reactions are inevitable consequences of such measures, especially among the vulnerable population. ${ }^{2}$ Along with these, people with substance use disorders face unique challenges like poor COVID-19 infection recovery and substance withdrawals. This unique period also has the potential for new addictive behaviours like video gaming and digital addictions.

People who smoke and vape tobacco may have diseases like chronic obstructive pulmonary disease (COPD) which can adversely affect recovery from COVID-19 infection. There is emerging evidence suggests that exposure to aerosols from E-cigarettes harms the cells of the lung, enhanced tissue damage and inflammation diminish the ability to respond to infections like influenza-like viruses. Frequent touching of the mouth while smoking increases the risk of infection. The Centre for Evidence-Based Medicine at the University of Oxford reported that smoking seemed to be a factor associated with poor survival in Italy, where $24 \%$ of people smoke. Ex-smokers with rapid healing of lung health after quitting smoking have lower chances of COVID-19 morbidity and mortality. ${ }^{6}$ However, a preliminary meta-analysis on Chinese patients contradicts the association between active smoking and an enhanced risk of progressing towards severe disease in COVID-19 infection. ${ }^{5}$ Hence there is a need for biologically plausible studies to correlate the observation of higher mortality and increased infective rates in male smokers.

People with alcohol dependency may have severe withdrawals due to restricted measures taken by governments, though it's time-limited. However, people can procure through the black market with higher prices, whereas some with homemade brewing. As such consumptions reports surfaced in media. Majority of them may quit because of less conducive environment and less peer influence resulted from social distancing. However, prospective studies are required to know alcohol use trajectory in public once restricted public health measures are lifted off.

The prospect of self-quarantine and other public health measures may also disrupt harm reduction activities like access to syringe services, medications, and other support needed by people with Opioid Use Disorder. On the other side, people with opioid dependence and people who inject drugs face novel challenges of missing their daily medicine. people on agonist treatment face crippling withdrawals due to lockdown. ${ }^{1}$ Whereas the availability of illicit opioids and drugs in society exists but in hiked prices of about 3 to 5 times than usual. People with opioid use disorder or who are on higher doses of opioids for medical reasons may face an additional risk of respiratory illness. ${ }^{4}$ Since opioids act in the brainstem to suppress the respiratory drive, their use not only puts the user at risk of life-threatening or fatal overdose, it may also cause a harmful decrease of oxygen in the blood (hypoxemia). ${ }^{7}$ These multiple or persistent states of hypoxemia can be damaging to the brain. However, the chronic respiratory disease is already known risk factor for increase overdose among people taking opioids and may diminish their lung capacity from COVID-19 Infection.

Another important area to look at is behavioural addictions like video gaming, internet or digital addictive behaviours. It takes an overwhelming toll on this generation because the pleasure sources are limited to indoor activities, people vest a substantial time while watching television and their electronic gadgets. In case of extension of lockdown, the binge-watching of television and excessive play with electronic gadgets are likely to persist which may later result in behavioural addictions. Since WHO also recommends the active video game use as a coping method to stay at home policy there are higher chances for the young generation to develop behavioural addictions, especially in developing countries.

Overall, other risks of people with substance use disorders include less access to health care, homelessness, and a greater likelihood for incarceration. Prevailing homelessness or incarceration can expose people to such environments where they are in close contact with others who might also be at higher risk for infections. Limited access to health care puts people with addiction at greater risk for many illnesses, if health infrastructure of any country is not pushed to their full capacity, people with addictive illnesses, who are already stigmatized and underserved by the healthcare system will experience even greater barriers to get treatment for COVID-19.Innovative modes of service delivery to the needy warranted at this 
testing time like mobile medical treatment services, mobile testing labs etc.

\section{Conflict of Interest}

None.

\section{References}

1. Alexander GC, Stoller KB, Haffajee RL, Saloner B. An epidemic in the midst of a pandemic: Opioid use disorder and COVID-19. Ann Intern Med. 2020; doi:10.7326/M20-1141.

2. Banerjee D. The COVID-19 outbreak: Crucial role the psychiatrists can play. Asian J Psychiatry. 2020;50:102014. doi: 10.1016/j.ajp.2020.102014.

3. Coronavirus [Internet]. World Health Organization. World Health Organization; [cited 2020May10]. Available from:https://www.who.int/emergencies/diseases/novelcoronavirus-2019

4. Leece P, Cavacuiti C, Macdonald EM, Gomes T, Kahan M, Srivastava A, et al. Canadian drug safety and effectiveness research network. Predictors of opioid-related death during methadone therapy. J Subst Abuse Treat. 2015;57:30-5.

5. Lippi G, Henry BM. Active smoking is not associated with severity of coronavirus disease 2019 (COVID-19). Eur J Intern Med. 2020;75:107-8.

6. Madison MC, Landers CT, Gu BH, Chang CY, Tung HY, You $\mathrm{R}$, et al. Electronic cigarettes disrupt lung lipid homeostasis and innate immunity independent of nicotine. J Clin Invest. 2019;129(10):4290-4304.

7. Volkow ND. Collision of the COVID-19 and Addiction Epidemics [Internet]. Ann Intern Med. American College of Physicians; 2020 [cited 2020May10]. Available from: https://www.ncbi.nlm.nih.gov/pubmed/32240293. DOI: https://doi.org/10.7326/M20-1212

How to cite this article: Dhagudu NK, Kumar EO. Covid-19 pandemic implications from an addiction perspective. Telangana J Psychiatry. 2020;6(1):102-103. 